Notre Dame Journal of Formal Logic

Volume 47, Number 4, 2006

\title{
The Embedding Theorem: Its Further Developments and Consequences. Part 1
}

\author{
Alexei Y. Muravitsky
}

\begin{abstract}
We outline the Gödel-McKinsey-Tarski Theorem on embedding of Intuitionistic Propositional Logic Int into modal logic $\mathbf{S 4}$ and further developments which led to the Generalized Embedding Theorem. The latter in turn opened a full-scale comparative exploration of lattices of the (normal) extensions of modal propositional logic $\mathbf{S 4}$, provability logic $\mathbf{G L}$, proof-intuitionistic logic KM, and others, including Int. The present paper is a contribution to this part of the research originated from the Gödel-McKinsey-Tarski Theorem. In particular, we show that the lattice ExtInt of intermediate logics is likely to be the only constructing block with which $\operatorname{Ext} \mathbf{S} \mathbf{4}$, the lattice of the extensions of $\mathbf{S 4}$, can be formed. We, however, advise the reader that our exposition is different from the historical lines along which some of the results discussed below came to light. Part 1, presented here, deals mostly with structural issues of extensions of logics, where algebraic semantics, though underlying this approach, is used merely occasionally. Part 2 will be devoted to algebraic analysis of the Embedding Theorem.
\end{abstract}

\section{Introduction}

The assertion on embedding of the intuitionistic propositional logic Int into modal logic in order to interpret the former in terms of the classical propositional logic $\mathbf{C l}$ and the notion of provability was conjectured in 1933 by Gödel in [5]. However, it gained the status of a theorem only in 1948 after the appearance in print of the paper [15] by McKinsey and Tarski. In his short note, Gödel proposed an operation which, applied to an assertoric formula, results in a modal formula so that, as Gödel suggested, the intuitionistic logic can be regarded through this operation as a fragment of modal logic S4. (See Theorem 2.1, Part 1.)

Received October 30, 2005; accepted May 15, 2006; printed December 28, 2006 2000 Mathematics Subject Classification: Primary, 03B45, 03B53

Keywords: modal logic, intermediate logic, McKinsey-Tarski translation, distributive lattice

(c)2006 University of Notre Dame 
In light of this Gödel-McKinsey-Tarski Embedding Theorem, a similar embedding of Int into a proper extension of S4, discovered by Grzegorczyk in the 1960s (see Theorem 2.1, Part 2), probably was not a big surprise at the time. The important role of Grzegorczyk's logic became clear almost a decade later when the attention of the propositional logic community turned from particular logics to the lattices of their extensions and especially to the comparative investigation of these lattices. The first work in this still growing field was the paper by Maksimova and Rybakov [13].

In the present paper we reconstruct the tree that is rooted in the Gödel-McKinseyTarski Embedding Theorem. In our exposition we emphasize logical dependences of things rather than the chronological order within which they were discovered. Also, we will not discuss semantics here, to which Part 2 will presumably be devoted. In particular, we intend to analyze there the algebraic constructions, used in the generalized theorem on embedding (Theorem 2.2) and bring other constructions into discussion.

Although having begun with the Gödel-McKinsey-Tarski Embedding Theorem, our own nonhistorical journey originates in Corollary 2.6 (Blok-Esakia inequality), which we regard as an indication that any normal extension of modal logic $\mathbf{S 4}$ can be seen as a twofold structure. ${ }^{1}$ The intention of a better understanding of this view is the underlying idea of the present work. As a result we get a new picture of the lattice of all normal extensions of $\mathbf{S 4}$.

\section{Starting Point}

We will be using two languages of propositional logic. One, called assertoric, contains logical connectives: $\wedge$ (conjunction), $\vee$ (disjunction), $\neg$ (negation), and $\rightarrow$ (material implication). Adding to this language modality $\square$, we get the modal propositional language. We use letters $A, B, \ldots$ to denote unspecified assertoric formulas and $\Sigma$ to denote a set (possibly empty) of such formulas, and we use letters $\alpha, \beta, \ldots$ for unspecified modal formulas and $\Gamma$ for a set (possibly empty) of modal formulas. In both languages we build up formulas over one and the same infinite set of propositional variables $p, q, \ldots$ (with or without indices).

We will need a mapping from the set of assertoric formulas into the set of modal formulas, defined as follows. Let $A^{\mathrm{t}}$ be the resulting modal formula by placement of $\square$ in front of every subformula of formula $A .^{2}$ We denote by $\Sigma^{\mathbf{t}}$ the resulting set of modal formulas when we apply this operation to all formulas in $\Sigma$.

Now we introduce into consideration the following propositional logics: Int, S4, and Grz, as well as the sets of their normal extensions, respectively, ExtInt, Ext S4, and ExtGrz. It is well known (cf., e.g., [2]) that the three last sets of logics are distributive lattices with respect to set intersection $\cap$ as the greatest lower bound and union of two logics, closed under the postulated rules of inference, as their least upper bound; we denote the latter operation by $\oplus$. We will be considering here only consistent normal extensions of a designated logic, that is, those which are closed under the rules of inference postulated in this logic. We use the following definitions of the logics mentioned above.

All three have the rule of (simultaneous) substitution and modus ponens, or $d e$ tachment, as their rules of inference-the only postulated rules for the assertoric logics in our consideration. In addition, $\mathbf{S 4}$ and $\mathbf{G r z}$, as well as their (normal) extensions, have also the rule of necessitation, which derives $\square \alpha$ from $\alpha$. 
The intuitionistic logic Int is defined by the same axiomatic system as in [10] except that we use substitution instead of axiom schemes. We will denote by Int $+\Sigma$ the extension of Int when $\Sigma$ is added to the axioms of Int. ${ }^{3}$ For example, the classical logic $\mathbf{C l}$ is Int $+\neg \neg p \rightarrow p$. The similar definition applies to forming extensions of modal logics. As usual, we write $A+B+C$ instead of $\{A, B, C\}$, and so forth, as well as $\alpha+\beta+\gamma$ instead of $\{\alpha, \beta, \gamma\}$, and so on.

Now let us consider $\mathbf{C l}$ in the modal language, endowed with the rule of necessitation, and denote this system by mCl. It will play a merely auxiliary part. So, following Gödel [5], we define

$$
\mathbf{S 4}=\mathbf{m C l}+\square(p \rightarrow q) \rightarrow(\square p \rightarrow \square q)+\square p \rightarrow p+\square p \rightarrow \square \square p .
$$

Furthermore, $\mathbf{G r z}=\mathbf{S 4}+\square(\square(p \rightarrow \square p) \rightarrow p) \rightarrow p$ is known as Grzegorczyk logic. ${ }^{4}$ The logic $\mathbf{S 4}+p \rightarrow \square p$ is known to be the only maximal consistent extension of Grz as well as of $\mathbf{S 4}$. We use $\mathbf{S 4}+\Gamma, \mathbf{G r z}+\Gamma$, and so forth to denote a consistent extension of $\mathbf{S 4}, \mathbf{G r z}$, respectively, or that of some other logics.

In [5] Gödel expressed a strong belief that the first equivalence of the following theorem is true.

Theorem 2.1 (on embedding) For every formula $A$, the following equivalences hold:

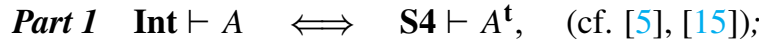

$$
\begin{aligned}
& \text { Part } 2 \text { Int } \vdash A \quad \Longleftrightarrow \quad \text { Grz } \vdash A^{\mathbf{t}} \quad \text { (cf. [8]). }
\end{aligned}
$$

This theorem was generalized in the 1970s as follows.

Theorem 2.2 (generalized on embedding, [15] + [12]) Let $M$ be a modal logic such that $\mathbf{S 4} \subseteq M \subseteq$ Grz. Then for any set $\Sigma$ of assertoric formulas and a formula A,

$$
\text { Int }+\Sigma \vdash A \Longleftrightarrow M+\Sigma^{\mathbf{t}} \vdash A^{\mathbf{t}} \text {. }
$$

The equivalence in Theorem 2.2 gives the grounds for the following definitions:

$$
\tau(\mathbf{I n t}+\Sigma)=\mathbf{S} \mathbf{4}+\Sigma^{\mathbf{t}} \text { and } \sigma(\mathbf{I n t}+\Sigma)=\mathbf{G r z}+\Sigma^{\mathbf{t}} .
$$

By virtue of Theorem 2.2, these definitions are correct. We show, for example, correctness of $\tau$.

Let us assume that an extension of Int can be axiomatized as Int $+\Sigma$ and Int $+\Sigma_{1}$. According to Theorem 2.1, each formula in $\Sigma_{1}$ is derivable in $\mathbf{S 4}+\Sigma^{\mathbf{t}}$ and each formula in $\Sigma$ is derivable in $\mathbf{S 4}+\Sigma_{1}^{\mathbf{t}}$; that is, $\mathbf{S 4}+\Sigma^{\mathbf{t}}=\mathbf{S 4}+\Sigma_{1}^{\mathbf{t}}$. In the same manner, one can show correctness of $\sigma$.

It follows from these definitions that

$$
\sigma(L)=\tau(L) \oplus \mathbf{G r z}
$$

for each assertoric $L \in \operatorname{ExtInt.}$

Two years before the general result of Theorem 2.2 on embedding had been established, Maksimova and Rybakov [13] had conducted a comparative investigation of lattices ExtInt and ExtS4. So they were the first to introduce mappings $\tau$ and $\sigma$, as well as the mapping $\rho: \operatorname{Ext} \mathbf{S} \mathbf{4} \longrightarrow \operatorname{ExtInt,~as~follows:~}$

$$
\rho(\mathbf{S} 4+\Gamma)=\left\{A \mid \mathbf{S} 4+\Gamma \vdash A^{\mathbf{t}}\right\} .
$$

Some important characteristics of these mappings can already be seen from the following. 
Theorem $2.3([13]+[11]) \quad \rho(\mathbf{S} \mathbf{4}+\Gamma)$ is an extension of $\mathbf{I n t}$; in other words, the equality $\rho(\mathbf{S} \mathbf{4}+\Gamma)=\mathbf{I n t}+\rho(\mathbf{S} \mathbf{4}+\Gamma)$ holds. Moreover, $\rho$ is an epimorphism and both $\tau$ and $\sigma$ are monomorphisms from ExtInt into Ext $\mathbf{S} \mathbf{4}$ and from ExtInt into Ext Grz, respectively.

In terms of $\tau, \sigma$, and $\rho$, Theorem 2.2 implies that, for every $L \in$ ExtInt,

$$
\rho \circ \tau(L)=L \text { and } \rho \circ \sigma(L)=L,
$$

which gives rise to the question whether the composites $\tau \circ \rho$ and $\sigma \circ \rho$ are the identity function, too. As to the former, the identity $\tau \circ \rho(M)=M$ is not in general true. For example, by Theorem 2.2, $\tau \circ \rho(\mathbf{G r z})=\tau(\mathbf{I n t})=\mathbf{S 4}$. The following theorem explains the situation.

Theorem 2.4 For any $M \in$ Ext $\mathbf{S 4}$, the equality $\tau \circ \rho(M)=M$ holds if and only if there is $L \in$ ExtInt such that $M=\tau(L)$.

Proof It suffices to notice that logic $\tau(L)$ satisfies the equality in question. Indeed, $\tau \circ \rho(\tau(L))=\tau(L)$ by virtue of the first equality in (2).

From Theorem 2.4, it follows immediately that Grz cannot be represented as $\tau(L)$. In other words, if we define

$$
\mathcal{L}=\{\tau(L) \mid L \in \operatorname{Ext} \mathbf{I n t}\},
$$

then $\mathbf{G r z} \notin \mathscr{L}$. According to Theorem 2.3, $\mathscr{L}$ is a sublattice of $E x t \mathbf{S} \mathbf{4}$ and isomorphic to ExtInt.

The composite $\sigma \circ \rho$ is also in general not the identity function, since $\sigma \circ \rho(\mathbf{S 4})=\mathbf{G r z}$. The interaction between $\tau, \sigma$, and $\rho$ is quite subtle as one can see from the following.

Theorem $2.5([13]+([1],[3])) \quad$ The equality $\rho^{-1}\left(\mathbf{I n t}+\Sigma^{\mathbf{t}}\right)=\left[\mathbf{S 4}+\Sigma^{\mathbf{t}}, \mathbf{G r z}+\Sigma^{\mathbf{t}}\right]$ holds; that is, $\rho^{-1}(L)=[\tau(L), \sigma(L)]$ for any $L \in$ ExtInt.

Theorems 2.3 and 2.5 imply immediately the following.

Corollary 2.6 (Blok-Esakia inequality) For every modal logic $M \in$ ExtS4, $\tau \circ \rho(M) \subseteq M \subseteq \sigma \circ \rho(M)$.

Since, by Equation (1), $\sigma(L)$ is an extension of Ext $\mathbf{G r z}$, it is natural to ask whether $\sigma \circ \rho(\mathbf{G r z}+\Gamma)=\mathbf{G r z}+\Gamma$. It is well known that the answer is positive.

Theorem $2.7([13]+([1],[3])+[11]) \quad$ The equality $\sigma \circ \rho(\mathbf{G r z}+\Gamma)=\mathbf{G r z}+\Gamma$ holds for all consistent extensions of Grz. Hence $\rho$, restricted to Ext Grz, and $\sigma$ are inverses of one another and, therefore, lattices ExtGrz and ExtInt are isomorphic.

Let us focus for a moment on the lattice $\mathcal{L}$. One can notice that its least element is S4 and its greatest element equals $\mathbf{S 4}+\square \neg \square \neg \square p \rightarrow \square p$, that is, $\mathbf{S 5}$.

We conclude this section with the following remark.

Remark 2.8 By Theorem 2.4, logic Grz cannot be defined as $\mathbf{S 4}+\Sigma^{\mathbf{t}}$ for any set $\Sigma$. In Section 4, we will prove that it remains to be true for all consistent extensions of Grz. On the other hand, each extension of Grz can be represented as $\mathbf{G r z}+\Sigma^{\mathbf{t}}$ for some $\Sigma$. (See Theorem 2.7 above.) We leave the question open whether $\mathbf{G r z}$ is the least logic in Ext $\mathbf{S} \mathbf{4}$ with this property. 


\section{Some Algebraic Requisites}

We will be using the notion of an S4-algebra, also known as topological Boolean algebra, ${ }^{6}$ interior algebra, ${ }^{7}$ closure algebra,${ }^{8}$ and topoboolean algebra, ${ }^{9}$ that is, a universal algebra $\langle\mathbf{B} ; \wedge, \vee, \neg, \mathbf{0}, \mathbf{1}, \square\rangle$, where $\langle\mathbf{B} ; \wedge, \vee, \neg, \mathbf{0}, \mathbf{1}\rangle$ is a Boolean algebra with the least element $\mathbf{0}$ and the greatest element $\mathbf{1}$. The first two operations, $\wedge$ and $\vee$, are binary operations of a greatest lower bound and a least upper bound, respectively, when the partial order $x \leqslant y$ is understood as $x \wedge y=x$ or $x \vee y=y$, and the operation $\neg$ is a unary operation of complement. The operation $\square$ is called an interior operation and is subject to the following identities:

$\square(x \wedge y)=\square x \wedge \square y, \quad \square x \wedge x=\square x, \quad \square \square x=\square x, \quad$ and $\quad \square \mathbf{1}=\mathbf{1}$,

valid for all $x$ and $y$ in $\mathbf{B}$.

Connection with logic arises when one considers varieties of $\mathbf{S 4}$-algebras, that is, such sets of $\mathbf{S 4 - a l g e b r a s ~ t h a t ~ a r e ~ d e t e r m i n e d ~ b y ~ i d e n t i t i e s ~ a d d i t i o n a l ~ t o ~ t h e ~ i d e n t i t i e s ~}$ above which determine the variety of all $\mathbf{S 4}$-algebras. All the varieties of $\mathbf{S 4}$-algebras form a lattice dually isomorphic to ExtS4. The latter, as it follows from Theorem 2 in [13], is a Heyting algebra ${ }^{10}$ and, hence, is distributive.

In this paper, we deal only with particular S4-algebras and postpone investigation of varieties to Part 2. We will be using distributivity of ExtS4 in Sections 9 and 10, referring to the distributivity test: A lattice is distributive if and only if it contains neither a diamond nor a pentagon as its sublattices. (See Theorem II.1 in [7].)

An element $a$ of an S4-algebra is called open if $\square a=a$. All open elements of an S4-algebra form a Heyting algebra with respect to the same $\leq$ relation as in the original S4-algebra. This Heyting algebra is called the skeleton of the given S4algebra. Generating the Boolean subalgebra by the skeleton of an S4-algebra, that is, using only Boolean operations of the signature of the latter, we get an $\mathbf{S 4}$-subalgebra of it, called Boolean-generated (or simply B-generated). Any B-generated algebra satisfies the identity

$$
\square(\neg \square(\neg x \vee \square x) \vee x)=\square x .
$$

An S4-algebra satisfying the last identity is called a Grzegorczyk algebra, or Grzalgebra for short. The variety of the Grz-algebras determines logic Grz. Thus, every B-generated algebra is a Grz-algebra and the converse is true for finite $\mathbf{S 4}$-algebras. Both observations are due to Maksimova [12]. They will be used occasionally without reference.

\section{Lattices ExtGrz and $\mathcal{L}$}

We can note that, by virtue of Theorem 2.7, any $M \in \operatorname{Ext} \mathbf{G r z}$ can be represented as $\sigma(L)$ for some $L \in \operatorname{Ext}$ Int. However, no $M \in \operatorname{Ext} \mathbf{G r z}$ can be represented as $\tau(L)$.

We want to note the following well-known observation.

Proposition 4.1 ([2], p. 93) Logic Grz, which is the least element in ExtGrz, and logic $\mathbf{S 4}+\square \neg \square \neg \square p \rightarrow \square p(=\mathbf{S 5})$, which is the greatest element in $\mathcal{L}$, are incomparable. Therefore, Ext Grz and $\mathcal{L}$ do not have common elements. ${ }^{11}$

Our next remark concerns lattice $\mathcal{L}$ and seems unimportant, but it allows us to introduce a new character into our discussion. Let us denote $M_{0}=\mathbf{G r z} \cap \mathbf{S 5}$. According to Theorem 1 in [13], $M_{0}=\mathbf{S 4}+(\square(\square(p \rightarrow \square p) \rightarrow p) \rightarrow p) \vee \square \neg \square \neg \square q \rightarrow \square q$. On the other hand, Grz $\oplus \mathbf{S 5}=\mathbf{G r z}+\square \neg \square \neg \square p \rightarrow \square p=\mathbf{S 4}+p \rightarrow \square p$. The last equality is true by virtue of Theorem 2.7. 
Proposition $4.2 \quad M_{0} \notin \mathscr{L}$.

Proof If it were otherwise, that is, $M_{0}=\tau(L)$, then we would have $\rho\left(M_{0}\right)=$ Int, since $M_{0} \in[\mathbf{S 4}, \mathbf{G r z}]$. On the other hand, $\rho\left(M_{0}\right)=\rho(\tau(L))=L$; that is, $L=$ Int, which implies $M_{0}=\mathbf{S 4}$. This is a contradiction, for the formula $(\square(\square(p \rightarrow \square p) \rightarrow p) \rightarrow p) \vee \square \neg \square \neg \square q \rightarrow \square q$ is not valid on an 8-element S4algebra with only three open elements: an atom, $\mathbf{0}$, and $\mathbf{1}$. As a refuting assignment, we can assign $p$ and $q$ any element that covers the open atom of the algebra.

According to Corollary 2.6 and Equation (1), for every modal logic $M, \tau \circ \rho(M)$ $\subseteq M \subseteq \mathbf{G r z} \oplus \tau \circ \rho(M)$. In other words, the equation

$$
M=M^{*} \oplus \tau \circ \rho(M)
$$

is solvable for $M^{*} \in[\mathbf{S 4}, \mathbf{G r z}]$. We will be preoccupied with this issue in the next section.

\section{$5 \quad \tau$-Representation for the Logics in ExtS4}

For any logic $M$, we call the right-hand side of the equality

$$
M=M^{*} \oplus \tau(L),
$$

where $M^{*} \in[\mathbf{S 4}, \mathbf{G r z}]$ and $L \in E x t \mathbf{I n t}$, a $\tau$-representation of $M$ with a modal component $M^{*}$ and an assertoric component $L$. It is clear that the assertoric component equals $\rho(M)$, since $\rho(M)=\rho\left(M^{*} \oplus \tau(L)\right)=\rho\left(M^{*}\right) \oplus \rho(\tau(L))=$ Int $\oplus L=L$. Thus each modal logic has a unique assertoric component of its $\tau$-representation, but its modal component may vary. However, given $M$, Equation (3) has always a solution for $M^{*}$.

Given modal $\operatorname{logic} M, \operatorname{logic} M^{*}=M \cap \mathbf{G r z}$ is a solution to Equation (3), since, by virtue of distributivity of $E x t \mathbf{S 4}$, Corollary 2.6, and (1), we get

$(M \cap \mathbf{G r z}) \oplus \tau \circ \rho(M)=(M \oplus \tau \circ \rho(M)) \cap(\mathbf{G r z} \oplus \tau \circ \rho(M))=M \cap \sigma \circ \rho(M)=M$.

We note that Equation (3) can have more than one solution. If, for instance, $M=\mathbf{S 5}$, both $M^{*}=\mathbf{S} \mathbf{4}$ and $M^{*}=M_{0}$ are solutions to this equation. In general, if $M \in \mathcal{L}$, then any logic in [S4, $M \cap \mathbf{G r z}]$ is a solution to (3). It is obvious that, given $M$, logic $M \cap \mathbf{G r z}$ is the greatest solution to (3). Thus, if $M \in \mathscr{L}$, the interval [S4, $M \cap \mathbf{G r z}$ ] consists of the solutions to (3). Also, we observe that, if $M \in[\mathbf{S 4}, \mathbf{G r z}]$, there is the only solution to (3), which is $M$ itself.

Given $M^{*} \in[\mathbf{S 4}, \mathbf{G r z}]$ and $L \in E x t \mathbf{I n t}$, we can define modal logic by (4). We call the $\tau$-representation of $M$ saturated if $M^{*}=M \cap \mathbf{G r z}$.

Proposition 5.1 The set of all modal components of a logic $M$ is a dense sublattice of Ext $\mathbf{S} \mathbf{4}$ with the greatest element $M \cap \mathbf{G r z}$.

Proof Suppose both $M_{1}^{*}$ and $M_{2}^{*}$ satisfy (4). Then, clearly, $M=\left(M_{1}^{*} \oplus M_{2}^{*}\right) \oplus \tau(L)$ and also, by distributivity,

$$
\left(M_{1}^{*} \cap M_{2}^{*}\right) \oplus \tau(L)=\left(M_{1}^{*} \oplus \tau(L)\right) \cap\left(M_{2}^{*} \oplus \tau(L)\right)=M \cap M=M .
$$

Now assume, in addition, that $M_{1}^{*} \subseteq M^{*} \subseteq M_{2}^{*}$. Obviously, $M=M_{1}^{*} \oplus \tau(L)$ $\subseteq M^{*} \oplus \tau(L)=M_{2}^{*} \oplus \tau(L)=M$. 
If the lattice of the modal components of $\tau$-representations of logic $M$ has the least element $M^{*}$, we call the representation $M=M^{*} \oplus \tau(L)$ minimal. We leave open the following problem.

Problem 5.2 Does every modal logic $M \in E x t \mathbf{S 4}$ possess a minimal $\tau$-representation?

Borrowing notation from [13], we denote a logic $\mathbf{S} \mathbf{4}+\Gamma$ by $[\Gamma]$. The following property is well known:

$$
\left[\Gamma_{1}\right] \oplus\left[\Gamma_{2}\right]=\left[\Gamma_{1} \cup \Gamma_{2}\right]
$$

see [13] for detail.

Proposition 5.3 Let $M^{*}$ be a modal component of logic $M$ given by its $\tau$ representation $M=M^{*} \oplus \tau(L)$. Then $\left[M^{*} \backslash \tau(L)\right]$ is also a modal component of $M$. Moreover, the process of "subtracting $\tau(L)$ " stabilizes after at most one step.

Proof Using (5), we get

$$
\left[M^{*} \backslash \tau(L)\right] \oplus \tau(L)=\left[\left(M^{*} \backslash \tau(L)\right) \cup \tau(L)\right]=\left[M^{*} \cup \tau(L)\right]=M^{*} \oplus \tau(L)=M .
$$

Now we will show that this process stabilizes. Indeed, let $M_{1}^{*}=\left[M^{*} \backslash \tau(L)\right]$. We have

$$
\left[M_{1}^{*} \backslash \tau(L)\right]=\left(M_{1}^{*} \backslash(\tau L)\right) \cup \Gamma\left[M_{1}^{*} \backslash \tau(L)\right]=\left(M_{1}^{*} \backslash \tau(L)\right) \cup \Gamma,
$$

for some $\Gamma \subseteq \tau(L)$. Then $\left[M_{1}^{*} \backslash \tau(L)\right] \backslash \tau(L)=\left(\left(M_{1}^{*} \backslash \tau(L)\right) \cup \Gamma\right) \backslash \tau(L)=M_{1}^{*} \backslash \tau(L)$.

Example 5.4 According to Proposition 4.1, $[\mathbf{G r z} \backslash \tau(L)]=\mathbf{G r z}$ for all $L$.

A $\tau$-representation $M=M^{*} \oplus \tau(L)$ is called fine if

$$
\left[M^{*} \backslash \tau(L)\right]=M^{*} \text {. }
$$

It is clear that any minimal $\tau$-representation must be fine. It is also clear that the $\tau$-representation of $\mathbf{G r z}$ is fine.

Problem 5.5 Is any $\tau$-representation of any consistent Grz $+\Gamma$ fine?

Now we want to consider the structure of the modal components in the fine $\tau$ representations of a logic.

Proposition 5.6 All modal components of a modal logic M satisfying (6) form a directed downward partially ordered set which is a join semilattice.

Proof Let us first prove that any two modal components of $M$ satisfying (6) have a lower bound satisfying (6). We remind the reader that the set of all modal components of $M$ is a lattice (Proposition 5.1). The conclusion, then, follows from the last observation and Proposition 5.3.

Now suppose that $M_{1}^{*}$ and $M_{2}^{*}$ are two distinct modal components of $M$ and both satisfy (6). Then we have

$$
\begin{aligned}
M_{1}^{*} \oplus M_{2}^{*} & =\left[M_{1}^{*} \backslash \tau(L)\right] \oplus\left[M_{2}^{*} \backslash \tau(L)\right] \\
& =\left[\left(M_{1}^{*} \backslash \tau(L)\right) \cup\left(M_{2}^{*} \backslash \tau(L)\right)\right] \\
& =\left[\left(M_{1}^{*} \cup M_{2}^{*}\right) \backslash \tau(L)\right] \\
& \subseteq\left[\left(M_{1}^{*} \oplus M_{2}^{*}\right) \backslash \tau(L)\right] \\
& \subseteq M_{1}^{*} \oplus M_{2}^{*} .
\end{aligned}
$$


Thus $M_{1}^{*} \oplus M_{2}^{*}$ satisfies (6).

\section{$6 \quad \tau_{0}$-Representations}

In this section, we focus exclusively on the extensions of the modal logic $M_{0}=\mathbf{G r z} \cap \mathbf{S 5}$. For any logic $L \in \operatorname{ExtInt}$, we define

$$
\tau_{0}(L)=M_{0} \oplus \tau(L) .
$$

It is obvious that

$$
\sigma(L)=\mathbf{G r z} \oplus \tau_{0}(L) .
$$

For each logic $M \in E x t M_{0}$, we call a $\tau_{0}$-representation the right side of the equation $M=M^{*} \oplus \rho \circ \tau(M)$, where $M^{*} \in \operatorname{Ext}_{0} \cap[\mathbf{S 4}, \mathbf{G r z}]$.

We notice that every modal logic $M \in E x t M_{0}$ has a $\tau_{0}$-representation. Indeed, let $M^{*}$ be a modal component of any $\tau$-representation of $M$; that is, $M=M^{*} \oplus \tau \circ \rho(M)$. Then, since $M_{0} \subseteq M$, we have $M=M_{0} \oplus M=$ $\left(M_{0} \oplus M^{*}\right) \oplus \tau \circ \rho(M)$ and $M_{0} \oplus M \in \operatorname{Ext}_{0}$.

We note here that the saturated $\tau$-representation of any logic from $\left[M_{0}, \mathbf{G r z}\right]$ is its $\tau_{0}$-representation, because for every $M \in E x t M_{0}, M_{0} \subseteq M \cap \mathbf{G r z} \subseteq$ Grz. Also, one can notice that for any $L \in E x t I n t$,

$$
\rho \circ \tau_{0}(L)=L,
$$

since $\rho\left(M_{0} \oplus \tau(L)\right)=\rho\left(M_{0}\right) \oplus \rho(\tau(L))=$ Int $\oplus L=L$.

Now we intend to show that each logic in ExtM $M_{0}$ possesses a unique $\tau_{0^{-}}$ representation. For this purpose, for any fixed assertoric logic $L \in E x t I n t$, we define two mappings-h : $M \longmapsto M \oplus \tau(L)$ for each $M \in\left[M_{0}, \mathbf{G r z}\right]$ and $\mathbf{g}: N \longmapsto N \cap \mathbf{G r z}$ for each $N \in\left[\tau_{0}(L), \sigma(L)\right]$. We are going to show that $\mathbf{h}$ and $\mathbf{g}$ are inverse lattice isomorphisms.

Indeed, by distributivity and Definitions (7) and (1), we get

$\mathbf{h}(\mathbf{g}(N))=(N \cap \mathbf{G r z}) \oplus \tau(L)=(N \oplus \tau(L)) \cap(\mathbf{G r z} \oplus \tau(L))=N \cap \sigma(L)=N$.

On the other hand, since $\tau$ is monotone, $\tau(L) \subseteq \tau(\mathbf{C l})=$ S5. The last inclusion implies $\mathbf{G r z} \cap \tau(L) \subseteq \mathbf{G r z} \cap \mathbf{S 5}=M_{0}$.

Now by virtue of distributivity and the last inclusion, we get

$\mathbf{g}(\mathbf{h}(M))=\mathbf{G r z} \cap(M \oplus \tau(L))=(\mathbf{G r z} \cap M) \oplus(\operatorname{Grz} \cap \tau(L))=M \oplus(\mathbf{G r z} \cap \tau(L))=M$.

Finally, we notice that $\mathbf{h}$ and $\mathbf{g}$ are monotone. Thus we have proved the following theorem.

Theorem 6.1 For each $L \in$ ExtInt, the lattices $\left[M_{0}\right.$, Grz $]$ and $\left[\tau_{0}(L), \sigma(L)\right]$ are isomorphic.

What is a structure of the intervals mentioned in Theorem 6.1? The answer gives the following corollary.

Corollary 6.2 All the intervals $[\tau(L), \sigma(L)]$, as well as $\left[M_{0}, \mathbf{G r z}\right]$, are linearly ordered and have type $1+\omega^{*}$.

Proof According to Theorem 6.1, these intervals are isomorphic to $\left[\tau_{0}(\mathbf{C l}), \sigma(\mathbf{C l})\right]$, that is, to $\left[M_{0} \oplus \mathbf{S 5}, \mathbf{G r z} \oplus \mathbf{S 5}\right]$, which can be read as $[\mathbf{S 5}, \mathbf{S 4}+p \rightarrow \square p]$. However, it is well known that the latter is linearly ordered and has type $1+\omega^{*}[17]$. 
Corollary 6.3 Every extension of $M_{0}$ has a unique $\tau_{0}$-representation which is the saturated $\tau$-representation of this extension.

Proof $\quad$ Let $M$ be in $\left[M_{0}, \mathbf{G r z}\right]$ and suppose $M=M_{1}^{*} \oplus \tau\left(L_{1}\right)=M_{2}^{*} \oplus \tau\left(L_{2}\right) ; M_{1}$ and $M_{2}$ lie also in $\left[M_{0}, \mathbf{G r z}\right]$. As we noted before, $L_{1}=L_{2}=\rho(M)$. On the other hand, the equality $M_{1}^{*} \oplus \tau(\rho(M))=M_{2}^{*} \oplus \tau(\rho(M))$ means that $\mathbf{h}\left(M_{1}^{*}\right)=\mathbf{h}\left(M_{2}^{*}\right)$; therefore, $M_{1}^{*}=M_{2}^{*}$.

Finally, as we noticed above in this section, the saturated $\tau$-representation of any logic from $\left[M_{0}, \mathbf{G r z}\right]$ is its $\tau_{0}$-representation.

Let $\left[M_{0}, \mathbf{G r z}\right]$ be ordered as follows:

$$
M_{0} \subset \cdots \subset M_{3} \subset M_{2} \subset M_{1}=\mathbf{G r z} .
$$

Corollary 6.4 Let $M \in[\mathbf{S 4}, \mathbf{G r z}]$ and $n \geqslant 1$. Then $M$ is a subset of $M_{n}$ but not a subset of $M_{n+1}$ if and only if $M \oplus M_{0}=M_{n}$.

Proof Suppose $M$ is included in $M_{n}$ but not in $M_{n+1}$. Obviously, $M \oplus M_{0} \subseteq M_{n}$. If this inclusion were proper, then, since $M \oplus M_{0} \in\left[M_{0}, \mathbf{G r z}\right]$ and by virtue of Corollary 2.6, the inclusion $M \oplus M_{0} \subseteq M_{n+1}$ would be true, which is a contradiction.

On the other hand, the equality $M \oplus M_{0}=M_{n}$ implies that $M \subseteq M_{n}$. If we also had the inclusion $M \subseteq M_{n+1}$, we would conclude that $M_{n} \subseteq M \subseteq M_{n+1}$. This is a contradiction.

\section{Partitioning Lattice $\operatorname{Ext}_{\mathbf{0}}$ in Slices and Layers}

For any $M_{n} \in\left[M_{0}, \mathbf{G r z}\right]$, we define

$$
\tau_{n}(L)=M_{n} \oplus \tau(L)
$$

as a function from $E x t I n t$ into $E x t M_{0}$. Then, for a fixed $n$, we call the set $\left\{\tau_{n}(L) \mid L \in\right.$ Ext Int $\}$ the $n$th slice of Ext $M_{0}$.

For a fixed $M_{n} \in\left[M_{0}, \mathbf{G r z}\right]$, we denote

$$
\tau_{n}(L)=M_{n} \oplus \tau(L) .
$$

Note that $\tau_{1}(L)=\sigma(L)$.

We remind the reader that, according to [17], all the extensions of S5 in Ext $\mathbf{S 4}$ are linearly ordered in type $1+\omega^{*}$ as follows:

$$
\mathbf{S 5}=S_{0} \subset \cdots \subset S_{3} \subset S_{2} \subset S_{1}=\mathbf{S 4}+p \rightarrow \square p .
$$

Proposition 7.1 For each $n \geqslant 0, S_{n}=M_{n} \oplus \mathbf{S 5}$ and $M_{n}=S_{n} \cap$ Grz.

Proof It is obvious that $S_{0}=\mathbf{S 5}=\mathbf{G r z} \cap \mathbf{S 5} \oplus \mathbf{S 5}=M_{0} \oplus \mathbf{S 5}$. Also, according to Proposition 4.2, we have $S_{1}=\mathbf{S 4}+p \rightarrow \square p=\mathbf{G r z} \oplus \mathbf{S 5}=M_{1} \oplus \mathbf{S 5}$.

Next we notice that each $M_{n} \oplus \mathbf{S 5}$ is an extension of $\mathbf{S 5}$, a $\tau_{0}$-representation of which $M_{k} \oplus \mathbf{S 5}$. Therefore, the proof will be completed when we show that the equality $M_{n} \oplus \mathbf{S 5}=M_{k} \oplus \mathbf{S} 5$ implies $n=k$. Since $\mathbf{S 5}=\tau(\mathbf{C l})$, this implication follows from Corollary 6.3.

Now the second equality of the statement follows easily from the first by distributivity. Indeed, $S_{n} \cap \mathbf{G r z}=\left(M_{n} \oplus \mathbf{S 5}\right) \cap \mathbf{G r z}=M_{n} \oplus M_{0}=M_{n}$.

Corollary 7.2 $\bigcap_{n \geqslant 1} M_{n}=M_{0}$. 
Proof The proof follows immediately from the second equality of Proposition 7.1.

Proposition 7.3 The mapping $\tau_{n}$ is a lattice isomorphism from ExtInt onto $\left[M_{n}, S_{n}\right]$.

Proof It is clear that $\tau_{n}$ is a lattice homomorphism. Also, $\tau_{n}$ is a one-one mapping. Indeed, $M_{n} \oplus \tau\left(L_{1}\right)=M_{n} \oplus \tau\left(L_{2}\right)$ implies $M_{n} \oplus \tau\left(L_{1}\right) \oplus \mathbf{G r z}=M_{n} \oplus \tau\left(L_{2}\right) \oplus \mathbf{G r z}$; that is, $\sigma\left(L_{1}\right)=\sigma\left(L_{2}\right)$. Therefore, by virtue of Theorem $2.7 L_{1}=L_{2}$.

Next we notice that $\tau_{n}(\mathbf{I n t})=M_{n}$ and $\tau_{n}(\mathbf{C l})=M_{n} \oplus \mathbf{S 5}=S_{n}$. Now assume that $M_{n} \subset M \subset S_{n}$ where neither $n=0$ nor $n=1$. According to Corollary 6.3, $M=M_{k} \oplus \tau(L)$, where $M_{k}=M \cap$ Grz. Therefore, $M_{n} \subseteq M_{k}$ and hence $n \geqslant k$. If it were the case that $n>k$, we would have $S_{k}=M_{k} \oplus \mathbf{S 5} \subseteq S_{n}$, since $M_{k} \subseteq S_{n}$ and $S_{n} \subset S_{k}$, that is, a contradiction. Thus $n=k$; hence, $M=\tau_{n}(L)$.

Theorem 7.4 All n-slices form a partition of ExtM0.

Proof Let $M \in E_{0 x t}$. If $M_{i} \subseteq M$ for all $i \geqslant 0$, then $M \in\left[M_{1}, S_{1}\right]=$ [Grz, S4 $+p \rightarrow \square p$ ]. If $M_{i} \nsubseteq M$ for all $i \geqslant 1$, then $M \in\left[M_{0}, S_{0}\right]=\left[M_{0}, \mathbf{S 5}\right]$.

Now we assume that $M_{n} \subseteq M$ and $M_{i} \nsubseteq M$ for all $i<n$ and $i \neq 0$. Thus $M \cap \mathbf{G r z}=M_{n}$ and, hence, $M_{n} \oplus \tau(\rho(M))$ is a $\tau_{0}$-representation of $M$. In other words, $M=\tau_{n}(\rho(M))$ and, according to Proposition 7.1, $M \in\left[M_{n}, S_{n}\right]$.

Next we prove that any two distinct slices are disjoint. Indeed, suppose $M \in\left[M_{n}, S_{n}\right] \cap\left[M_{k}, S_{k}\right]$ and $n<k$. If $n=0$, then $M_{0} \subset M_{k} \subseteq$ Grz $\cap \mathbf{S 5}=M_{0}$. So we assume that $1 \leqslant n<k$. Thus $M_{k} \subset M_{n}$ and, correspondingly, $S_{k} \subset S_{n}$. However, since $M_{n} \subseteq S_{k}, M_{n} \oplus \mathbf{S 5} \subseteq S_{k}$.

Proposition 7.5 For any $n \geqslant 1$, the $n$th slice and $\mathcal{L}$ do not intersect. ${ }^{12}$

Proof Suppose $M_{n} \subseteq M \subseteq \mathbf{S 5}$. Then $M_{n} \oplus \mathbf{S 5}=\mathbf{S 5}$. This contradicts Proposition 7.1 .

Problem 7.6 The 0th slice has at least one common logic with $\mathcal{L}$ - the logic S5. Do they have more common elements?

For a fixed $L \in \operatorname{Ext} \mathbf{I n t}$, we call $\left\{\tau_{n}(L) \mid n \geqslant 0\right\}$ an $L$-layer. We notice that $\tau_{n}(L)$ belongs to the $n$th slice. Therefore, by Proposition 7.5 , each $L$-layer has a linear order of type $1+\omega^{*}$ as follows:

$$
\tau_{0}(L) \subset \cdots \subset \tau_{3}(L) \subset \tau_{2}(L) \subset \tau_{1}(L) .
$$

We also notice that for every $n \geqslant 0$ and $L \in \operatorname{Ext} \mathbf{I n t}$,

$$
\rho\left(\tau_{n}(L)\right)=L .
$$

Let $\rho_{0}$ be the restriction of $\rho$ to $\operatorname{Ext} M_{0}$. It is clear that $\rho_{0}$ is an epimorphism from ExtM $_{0}$ onto ExtInt.

Lemma 7.7 For every $L \in \operatorname{ExtInt,}$

$$
\rho_{0}^{-1}(L)=\left[\tau_{0}(L), \tau_{1}(L)\right] .
$$


Proof Indeed,

$$
\begin{aligned}
\rho_{0}^{-1}(L) & =\left\{M \mid M \in \operatorname{Ext}_{0}, \rho(M)=L\right\} \\
& =\left\{M \mid M \in \operatorname{Ext} \mathbf{S 4}, M=M_{0} \oplus M, \rho(M)=L\right\} \\
& =\left\{M_{0} \oplus M \mid M \in \operatorname{Ext} \mathbf{S 4}, \rho(M)=L\right\} \\
& =\left\{M_{0} \oplus M \mid M \in \operatorname{Ext} \mathbf{S 4}, M \in \rho^{-1}(L)\right\} \\
& =\left\{M_{0} \oplus M \mid M \in \operatorname{Ext} \mathbf{S 4}, M \in[\tau(L), \sigma(L)]\right\} \text { by Theorem 2.5) } \\
& =\left\{M_{0} \oplus M \mid M \in \operatorname{Ext} \mathbf{S 4}, \tau(L) \subseteq M \subseteq \sigma(L)\right\} \\
& =\left\{M \mid M \in \operatorname{Ext}_{0}, \tau_{0}(L) \subseteq M \subseteq \sigma(L)\right\} \\
& =\left\{M \mid M \in \operatorname{Ext}_{0}, \tau_{0}(L) \subseteq M \subseteq \tau_{1}(L)\right\} .
\end{aligned}
$$

Proposition 7.8 For each $L \in \operatorname{Ext} \mathbf{I n t}, \rho_{0}^{-1}(L)$ is the L-layer.

Proof It follows from Lemma 7.7 that $\left\{\tau_{n}(L) \mid n \geqslant 0\right\} \subseteq \rho_{0}^{-1}(L)$. On the other hand, if $M \in \rho_{0}^{-1}(L)$, that is, $\tau_{0}(L) \subseteq M \subseteq \tau_{1}(L)$, then, first, by Equation (9), $L \subseteq \rho(M) \subseteq L$; that is, $\rho(M)=L$. Second, according to Corollary 6.3, there is $n \geqslant 0$ so that $M=M_{n} \oplus \tau(L)$; that is, $M=\tau_{n}(L)$.

One can see with the help of Proposition 7.8 that all the $L$-layers form a quotient lattice of $\operatorname{Ext}_{0}$, isomorphic to ExtInt.

\section{Modal Components of $\tau$-Representations of Logics in ExtM}

The following theorem holds.

Theorem 8.1 Let $M \in E^{2 x t} M_{0}$ and $M=M^{*} \oplus \tau(L)$ be an arbitrary $\tau$ representation of $M$. Then either there is a natural number $n \geqslant 1$ such that $M^{*} \subseteq M_{n}$ but $M^{*} \nsubseteq M_{n+p}$, for all $p \geqslant 1$, in which case $M$ belongs to the $n t h$ slice, or $M^{*}$ is included in all $M_{n}, n \geqslant 1$, in which case $M$ belongs to the 0th slice. Conversely, if $M$ belongs the nth slice for some $n \geqslant 1$, then $M^{*} \subseteq M_{n}$ but $M^{*} \nsubseteq M_{n+p}$ for all $p \geqslant 1$. If $M$ lies in the 0th slice, then $M^{*} \subseteq M_{0}$.

Proof Let $M=M_{k} \oplus \tau(L)$ be the $\tau_{0}$-representation of $M$ which, according to Corollary 6.3, is also its saturated $\tau$-representation. Therefore, we have $M^{*} \subseteq M_{k}$. Suppose $M^{*} \subseteq M_{n}$ but $M^{*} \nsubseteq M_{n+p}$ for all $p \geqslant 1$. It is clear that $M \subseteq M_{n} \oplus \tau(L)$. Therefore, $M_{k} \oplus \tau(L) \subseteq M_{n} \oplus \tau(L)$. On the other hand, $1 \leqslant k \leqslant n$. Therefore, $M_{n} \subseteq M_{k}$ and, hence, $M_{n} \oplus \tau(L) \subseteq M_{k} \oplus \tau(L)$. Thus $M_{k} \oplus \tau(L)=M_{n} \oplus \tau(L)$, which, by Corollary 6.3, implies that $M_{k}=M_{n}$; that is, $M$ belongs to the $n$th slice.

It is obvious that if $M^{*} \subseteq M_{n}$ for all $n \geqslant 1$, then $M^{*} \subseteq M_{0}$ and, hence, $M^{*} \oplus \tau(L) \subseteq M_{0} \oplus \tau(L)$; that is, $M_{k} \oplus \tau(L) \subseteq M_{0} \oplus \tau(L)$. On the other hand, the inclusion $M_{0} \oplus \tau(L) \subseteq M_{k} \oplus \tau(L)$ always holds. Therefore, we have $M_{k}=M_{0}$; that is, $M$ belongs to the 0th slice.

Now assume that $M$ belongs to an $n$th slice where $n \geqslant 1$ and $M=M^{*} \oplus \tau(L)$ is its $\tau$-representation. Then also $M=M_{n} \oplus \tau(L)$ where the latter is the saturated $\tau$-representation of $M$. Therefore, $M^{*} \subseteq M_{n}$. At the same time, if the inclusion $M^{*} \subseteq M_{n+1}$ were true, then according to the first part of the proof, $M$ would belong either to the 0 th slice or to a $k$ th slice with $k>n$. However, both conclusions would contradict Theorem 7.4. 
Corollary 8.2 Let $M$ belong to Ext $M_{0}$ and lie in the nth slice, $n \geqslant 1$. Also, let $M_{n}$ be the modal component of the $\tau_{0}$-representation of $M$. Then for every $M^{*} \in[\mathbf{S 4}, \mathbf{G r z}]$ such that $M^{*} \subseteq M_{n}$ and $M^{*} \nsubseteq M_{n+1}, M^{*} \oplus M_{0}=M_{n}$. On the other hand, if $M^{*}$ is any modal component of the $\tau$-representation of $M$, then $M^{*} \oplus M_{0}=M_{n}$; that is, $M^{*} \oplus M_{0}$ is the modal component of its saturated $\tau$ representation.

Proof The first statement follows immediately from Corollary 6.4. To prove the second statement, we apply Theorem 8.1 and Corollary 6.4 again.

We conclude this section with an example of a logic having two modal components.

Example 8.3 Logic $\mathbf{S 4}+p \rightarrow \square p$ has Grz and $\mathbf{S 4 . 1}$ among its modal components.

We recall that $\mathbf{S 4}+p \rightarrow \square p=S_{1}$ and $\mathbf{G r z}=M_{1}$. According to Proposition 7.1, $S_{1}=M_{1} \oplus \mathbf{S 5}$; that is, $S_{1}=M_{1} \oplus \tau(\mathbf{C l})$.

For another modal component we have to find such a proper sublogic of Grz that would not be included in $M_{2}$ or at least not be included in $S_{2}$-the logic of a 4element $\mathbf{S 4}$-algebra with only two open elements $-\mathbf{0}$ and $\mathbf{1}$. For such a candidate we can try $\mathbf{S 4}+\square \diamond p \rightarrow \diamond \square p$, that is, $\mathbf{S 4 . 1}$, since the latter is not valid on that algebra. However, $\mathbf{S 4 . 1} \oplus \mathbf{S 5}=S_{1}$. We also know that $\mathbf{S 4 . 1} \subset \mathbf{~ G r z}$.

\section{Partitioning the Interval [S4, Grz]}

We define the partition of the interval $[\mathbf{S 4}, \mathbf{G r z}]$ as follows. Let $\varepsilon_{0}$ be $\left[\mathbf{S 4}, M_{0}\right]$ and each $\mathcal{E}_{n}, n \geqslant 1$ consist of the logics that are included in $M_{n}$ but not in $M_{n+1}$. In view of Corollary 7.2, the family $\left\{\boldsymbol{E}_{n}\right\}_{n \geqslant 0}$ is a partition of $[\mathbf{S 4}, \mathbf{G r z}]$.

Theorem 9.1 Each $\mathcal{E}_{n}, n \geqslant 0$ is an interval.

Proof This is obvious for $\varepsilon_{0}$. Next we consider an $\varepsilon_{n}$, where $n \geqslant 1$. First we show that $\varepsilon_{n}$ is a sublattice. It is clear that $\varepsilon_{n}$ is closed under $\oplus$. Now we prove by contradiction that $\varepsilon_{n}$ is closed under $\cap$.

Let us suppose that there are two incomparable $\operatorname{logics} M$ and $N$ in $\mathcal{E}_{n}$ so that the inclusion $M \cap N \subset M_{n+1}$ holds. $^{13}$

Case $1 \quad M \oplus N=M_{n}$. It is clear that both inclusions $M \cap N \subseteq M \cap M_{n+1}$ and $M \cap N \subseteq N \cap M_{n+1}$ hold.

Case 1a In addition, we can assume that $M \cap N=M \cap M_{n+1}=N \cap M_{n+1}$ in which case $M, N, M_{n}, M_{n+1}$, and $M \cap N$ form a diamond. This is a contradiction.

Case 1b Now, in addition, we assume that either $M \cap N \subset M \cap M_{n+1}$ or $M \cap N \subset M_{n+1}$ holds. Let us consider the former. Then $M, M_{n}, N, M \cap N$, and $M \cap M_{n+1}$ form a pentagon. Again we arrive at a contradiction. In case when the other inclusion holds, Case 1c can be considered similarly.

Case $2 \quad M \oplus N \subset M_{n}$.

Case 2a In addition, we assume that $M \cap M_{n+1}=N \cap M_{n+1}$ which must be equal to $M \cap N$. Then $M, M \oplus N, M_{n}, M_{n+1}$, and $M \cap N$ form a pentagon.

Case 2b In addition, we assume now that $M \cap M_{n+1}=M \cap N$ but $M \cap N$ $\subset N \cap M_{n+1}$. Then $M, M \oplus N, N, N \cap M_{n+1}$, and $M \cap N$ form a pentagon. 
Case 2c When we have $N \cap M_{n+1}=M \cap N$ but $M \cap N \subset M \cap M_{n+1}$, then $M, M \oplus N, N, M \cap N$, and $M \cap M_{n+1}$ form a pentagon.

Case 2d In addition, we have $M \cap N \subset M \cap M_{n+1}$ and $M \cap N \subset N \cap M_{n+1}$. Since

$$
M \oplus\left(N \cap M_{n+1}\right)=(M \oplus N) \cap\left(M \oplus M_{n+1}\right)=(M \oplus N) \cap M_{n}=M \oplus N,
$$

$M, M \oplus N, N, N \cap M_{n+1}$, and $M \cap N$ form a pentagon. So we have a contradiction again.

Next we show that $\varepsilon_{n}$ satisfies the condition of Zorn's Lemma with respect to the inverse of the inclusion relation $\subseteq .{ }^{14}$ First we remind the reader that, by virtue of [13], Theorem 2, ExtS4 is a Heyting algebra. Let us consider a chain $\left\{N_{i}\right\}_{i \in I}$ of logics from $\varepsilon_{n}$ and let $N=\bigcap_{i \in I} N_{i}$. Each logic $N_{i}$ and $N$ can be regarded as a clopen in a compact topological space, namely, in Esakia space. ${ }^{15}$ Thus, if $N \subseteq M_{n+1}$, then $N_{i} \subseteq M_{n+1}$ for some $i \in I$, which is a contradiction. So, according to Zorn's Lemma, $\mathcal{E}_{n}$ has a minimal element with respect to $\subseteq$. Let us call this logic $K_{n}$. However, $K_{n}$ is not a merely minimal logic in $\mathcal{E}_{n}$, but the least one, since $\mathcal{E}_{n}$ is a sublattice. Logic $K_{n}$ is obviously included in $M_{n}$ but not in $M_{n+1}$ and, hence, each logic in the interval $\left[K_{n}, M_{n}\right]$ enjoys this property.

Problem 9.2 Is each logic $K_{n}$ finitely axiomatizable?

Problem 9.3 Is each interval $\left[K_{n}, M_{n}\right]$ isomorphic to ExtInt?

\section{The Common Part of the Modal Component and $\tau$-Component of an Arbitrary Logic from Ext $M_{0}$}

Let $M \in \operatorname{Ext}_{0}$ and we assume that $M=M^{*} \oplus \tau(L)$ is a $\tau$-representation of $M$. We define $\triangle M=M^{*} \cap \tau(L)$-the common part of the modal component and $\tau$-component of $M$. One can notice that $\mathbf{S 4} \subseteq \Delta M \subseteq M_{0}$; that is, $\triangle M \in \varepsilon_{0}$. In order to prove it we recall that $M^{*} \subseteq M_{n}$ for some $n \geqslant 0$ and $\tau(L) \subseteq$ S5. Therefore, $\triangle M \subseteq M_{1} \cap \mathbf{S 5}=M_{0}$. From the definition above we also notice that $\triangle M \subseteq M^{*} \cap M_{0}$.

Theorem 10.1 Let $M$ in Ext $M_{0}$ have a $\tau$-representation $M=M^{*} \oplus \tau(L)$. Then $\triangle M=M^{*} \cap M_{0}$ if and only if $M_{0} \subseteq \tau(L) .{ }^{16}$

Proof Suppose $M_{0} \subseteq \tau(L)$. This implies that $\triangle M \subseteq M^{*} \cap M_{0} \subseteq M^{*} \cap \tau(L)=\triangle M$. Conversely, assume that $\triangle M=M^{*} \cap M_{0}$ and $M$ belongs to the $n$th slice; that is, $M_{n}$ is the greatest modal component of $M$. We know that $M^{*} \subseteq M_{n}$. So we have two cases to consider.

Case $1 \quad M^{*}=M_{n}$. Then $M_{n} \cap \tau(L)=\triangle M=M_{n} \cap M_{0}=M_{0}$. It remains to notice that

$$
\begin{aligned}
M_{n} \cap \tau(L)=M_{0} & \left.\Longleftrightarrow M_{n} \cap \tau(\mathbf{C l}) \cap \tau(L)=M_{0} \quad \text { (by monotonicity of } \tau\right) \\
& \Longleftrightarrow M_{n} \cap \mathbf{S 5} \cap \tau(L)=M_{0} \\
& \Longleftrightarrow M_{0} \cap \tau(L)=M_{0} \quad \text { (since } M_{n} \subseteq M_{1} \text { and } M_{1} \cap \mathbf{S 5}=M_{0} \text { ) } \\
& \Longleftrightarrow M_{0} \subseteq \tau(L) .
\end{aligned}
$$


Case $2 M^{*} \subset M_{n}$. We first notice that the inclusion $\tau(L) \subseteq M_{n}$ cannot be true, for we would have, by virtue of monotonicity of $\rho$ and (2), that $L=$ Int. But then $\triangle M=\mathbf{S} 4$ and, hence, $M=M^{*} \subset M_{n}=M$, a contradiction. If, on the other hand, $M_{n} \subseteq \tau(L)$, then, the more so, $M_{0} \subseteq \tau(L)$. Thus, we will count that the logics $M_{n}$ and $\tau(L)$ are incomparable.

Next we show that $M^{*}$ and $\tau(L)$ are also incomparable. It is clear that $\tau(L) \nsubseteq M^{*}$, since $M^{*} \subseteq M_{n}$. For contradiction, we assume that $M^{*} \subseteq \tau(L)$. Then we have $M=\tau(L)$. But we have just proved that $M_{n}$ and $\tau(L)$ are incomparable, which implies that $\tau(L) \subset M_{n} \oplus \tau(L)=M$, a contradiction.

Next we notice that $M^{*} \cap \tau(L) \subset M_{n} \cap \tau(L)$, for in case of equality we would have to conclude that the pentagon with the vertices $M^{*}, M_{n}, M, \tau(L), M_{n} \cap \tau(L)$ is a sublattice of $\operatorname{Ext} \mathbf{S 4}$. Since $M_{n} \subseteq M_{1}$ and $\tau(L) \subseteq \mathbf{S 5}$, we have $M_{n} \cap \tau(L) \subseteq M_{0}$. Now we intend to prove that this inclusion cannot be proper. Indeed, assume for contradiction that $M_{n} \cap \tau(L) \subset M_{0}$. We notice that

$$
M^{*} \oplus\left(M_{n} \cap \tau(L)\right)=\left(M^{*} \oplus M_{n}\right) \cap\left(M^{*} \oplus \tau(L)\right)=M_{n} \cap M=M_{n} .
$$

Also, according to Theorem 8.1 and Corollary 6.4, $M_{0} \oplus M^{*}=M_{n}$. Thus, we found the pentagon with vertices $M^{*}, M_{n}, M_{0}, M_{n} \cap \tau(L)$, and $M^{*} \cap \tau(L)$. Therefore, $M_{n} \cap \tau(L)=M_{0}$, which, as we have proved above, is equivalent to the inclusion $M_{0} \subseteq \tau(L)$.

\section{Conclusion}

As the reader recalls, we started with the theorem on embedding of the intuitionistic propositional logic into the modal logic $\mathbf{S 4}$. This led us to the lattice $\mathscr{L}$ isomorphic to ExtInt. As we have seen, the last lattice serves as a main constructing block in forming the lattice ExtS4. We add below several remarks to this conclusion.

Remark 11.1 Consider the interval $\left[M_{0}, \mathbf{G r z}\right]$ in ExtS4. The filter generated by this interval equals $E x t M_{0}$, which in turn consists of the intervals $\left[M_{n}, S_{n}\right]$ each of which is a lattice replica of ExtInt. On the other hand, the ideal generated by $\left[M_{0}, \mathbf{G r z}\right]$ equals [S4, Grz], which consists of the intervals $\left[K_{n}, M_{n}\right]$.

Remark 11.2 The lattice $\mathcal{L}$ has at least two common points with the filter and ideal - the logic S5 with the filter and the logic $\mathbf{S 4}$ with the ideal.

In conclusion, we formulate the following conjectures.

Conjecture 11.3 The lattice Ext $\mathbf{S} \mathbf{4}$ consists of the filter and ideal as well as the lattice $\mathcal{L}$; there is nothing more in it.

Conjecture 11.4 The lattice $\mathcal{L}$ has only two common elements with the filter and ideal-S5 and S4, respectively.

Conjecture 11.5 Each interval $\left[K_{n}, M_{n}\right]$ is isomorphic to ExtInt.

\section{Notes}

1. This will be explained in Section 5 .

2. The operation $A \mapsto A^{\mathbf{t}}$ is often called the McKinsey-Tarski translation. 
3. If $\Sigma$ is nonempty, it must contain only classical tautologies in order to have Int $+\Sigma$ consistent.

4. Logic Grz was defined by Grzegorczyk in [8] and was named after him. The original definition contained a formula with two variables. The present definition is due to Maksimova [12].

5. The equality $\mathbf{S 4}+\square \neg \square \neg \square p \longrightarrow \square p=\mathbf{S 5}$ can be traced in [9].

6. See Rasiowa and Sikorski [16].

7. See [2], though Blok was the first to introduce it in his doctoral dissertation [1].

8. See McKinsey and Tarski [14].

9. This term was introduced in [11].

10. Another term for a Heyting algebra is a pseudo Boolean algebra. See Rasiowa and Sikorski [16].

11. We will prove a stronger statement in Section 7.

12. This proposition is a generalization of Proposition 4.1.

13. We note that the equality $M \cap N=M_{n+1}$ is impossible.

14. Cf. [6], Chapter 0, §4.

15. Esakia showed in [4], Chapter III, that any Heyting algebra can be embedded into the Heyting algebra of the open sets of a 0-dimensional, Hausdorff, compact topological space, which is defined as follows: the points are the prime filters of a given Heyting algebra and the topology is defined through its subbase- the images and their complements with respect to Stone embedding (Esakia space).

16. By Proposition 4.2, this improper inclusion can be replaced with the proper one.

\section{References}

[1] Blok, W. J., Varieties of Interior Algebras, Ph.D. thesis, University of Amsterdam, 1976. 528,539

[2] Chagrov, A., and M. Zakharyaschev, Modal Logic, vol. 35 of Oxford Logic Guides, The Clarendon Press, New York, 1997. Zbl 0871.03007. MR 1464942. 526, 529, 539

[3] Esakia, L. L., "On modal counterparts of superintuitionistic logics," pp. 135-36 in The Seventh All-Soviet Symposium on Logic and Methodology of Science, Abstracts, 1976. In Russian. 528

[4] Esakia, L. L., Algebry Geitinga. I. Teoriya Dvoistvennosti. (Heyting Algebras I: Dual Theory), Metsniereba, Tbilisi, 1985. MR 847050. 539 
[5] Gödel, K., "An interpretation of the intuitionistic propositional calculus," pp. 301-2 in Collected Works. Vol. I. Publications 1929-1936, edited by S. Feferman et al., The Clarendon Press, New York, 1986. Originally published as "Eine Interpretation des intuitionistischen Aussagenkalküls," pp. 39-40 in Ergebnisse eines mathematischen Kolloquiums, 4, 1933. Zbl 0592.01035. MR 831941. 525, 527

[6] Grätzer, G., Universal Algebra, 2d edition, Springer-Verlag, New York, 1979. Zbl 0412.08001. MR 538623. 539

[7] Grätzer, G., General Lattice Theory, 2d edition, Birkhäuser Verlag, Basel, 1998. Zbl 0909.06002. MR 1670580. 529

[8] Grzegorczyk, A., "Some relational systems and the associated topological spaces," Fundamenta Mathematicae, vol. 60 (1967), pp. 223-31. Zbl 0207.29603. MR 0218216. 527,539

[9] Hughes, G. E., and M. J. Cresswell, An Introduction to Modal Logic, Methuen and Co., Ltd., London, 1968. Zbl 0205.00503. MR 0439586. 539

[10] Kleene, S. C., Introduction to Metamathematics, D. Van Nostrand Co., Inc., New York, 1952. Zbl 0047.00703. MR 0051790. 527

[11] Kuznetsov, A. V., and A. Y. Muravitsky, "On superintuitionistic logics as fragments of proof logic extensions," Studia Logica, vol. 45 (1986), pp. 77-99. Zbl 0623.03027. MR 877303. 528, 539

[12] Maksimova, L. L., "Modal logics of finite layers," Algebra i Logika, vol. 14 (1975), pp. 304-19, 369. MR 0429488. 527, 529, 539

[13] Maksimova, L. L., and V. V. Rybakov, "The lattice of normal modal logics," Algebra i Logika, vol. 13 (1974), pp. 188-216, 235. Zbl 0315.02027. MR 0363810. 526, 527, 528, 529, 531, 537

[14] McKinsey, J. C. C., and A. Tarski, "The algebra of topology," Annals of Mathematics. Second Series, vol. 45 (1944), pp. 141-91. Zbl 0060.06206. MR 0009842. 539

[15] McKinsey, J. C. C., and A. Tarski, "Some theorems about the sentential calculi of Lewis and Heyting," The Journal of Symbolic Logic, vol. 13 (1948), pp. 1-15. Zbl 0037.29409. MR 0024396. 525, 527

[16] Rasiowa, H., and R. Sikorski, The Mathematics of Metamathematics, vol. 41 of Polska Akademia Nauk Monografie Matematyczne, Państwowe Wydawnictwo Naukowe, Warzawa, 1963. Zbl 0122.24311. MR 0163850. 539

[17] Scroggs, S. J., "Extensions of the Lewis system S5," The Journal of Symbolic Logic, vol. 16 (1951), pp. 112-120. Zb1 0043.00804. MR 0042352. 532, 533

Louisiana Scholars' College

Northwestern State University

Natchitoches LA 71497

alexeim@nsula.edu 